\title{
First Report of Bovine Leukemia Virus Infection in Yaks (Bos mutus) in China
}

\author{
Jian-Gang Ma,,2 Wen-Bin Zheng, ${ }^{1,2}$ Dong-Hui Zhou, ${ }^{2}$ Si-Yuan Qin, ${ }^{3}$ \\ Ming-Yang Yin, ${ }^{2}$ Xing-Quan $\mathrm{Zhu},{ }^{2}$ and Gui-Xue $\mathrm{Hu}^{1}$ \\ ${ }^{1}$ College of Animal Science and Technology, Jilin Agricultural University, Changchun, Jilin 130118, China \\ ${ }^{2}$ State Key Laboratory of Veterinary Etiological Biology, Key Laboratory of Veterinary Parasitology of Gansu Province, \\ Lanzhou Veterinary Research Institute, Chinese Academy of Agricultural Sciences, Lanzhou, Gansu 730046, China \\ ${ }^{3}$ General Station for Surveillance of Wildlife Diseases \& Wildlife Borne Diseases, State Forestry Administration (SFA), \\ Shenyang 110034, China
}

Correspondence should be addressed to Xing-Quan Zhu; zhuxingquan@caas.cn and Gui-Xue Hu; huguixue901103@163.com

Received 28 February 2016; Revised 7 May 2016; Accepted 16 May 2016

Academic Editor: Leon Spicer

Copyright (c) 2016 Jian-Gang Ma et al. This is an open access article distributed under the Creative Commons Attribution License, which permits unrestricted use, distribution, and reproduction in any medium, provided the original work is properly cited.

Enzootic bovine leukosis (EBL) is a chronic lymphosarcoma disease of cattle caused by bovine leukemia virus (BLV). No information is available concerning the epidemiology of BLV infection in yaks (Bos mutus). One thousand five hundred and eightyfour serum samples from 610 black yaks and 974 white yaks from Gansu province, northwest China, were collected between April 2013 and March 2014 and tested for BLV antibodies using a commercially available ELISA kit. The overall BLV seroprevalence in yaks was $21.09 \%(334 / 1584)$, with $24.26 \%$ (148/610) black yaks and $19.10 \%(186 / 974)$ white yaks yielding positive results. Risk factor analysis indicated that with the exception of breed $(\mathrm{OR}=1.36,95 \% \mathrm{CI}=1.06-1.73, P<0.05)$, the age, region, gender, farm, and the numbers of pregnancies were not considered as risk factors for the presence of BLV in yaks included in this study. This is the first report of BLV infection in yaks in China, which provides information for controlling BLV infection in yaks.

\section{Introduction}

Enzootic bovine leukosis (EBL) is a chronic lymphosarcoma disease of cattle caused by bovine leukemia virus (BLV), a member of the genus Deltaretrovirus in the family Retroviridae [1]. Transmission of BLV can occur through two routes: horizontal transmission (e.g., iatrogenic) and vertical transmission $[2,3]$. BLV infections may present as aleukemic (asymptomatic animals), persistent lymphocytosis (with hematological abnormalities), and lymphosarcoma (tumors), and most infections are subclinical. Approximately $30 \%$ of BLV infected cattle have persistent lymphocytosis, and malignant lymphosarcoma occurs in less than $5 \%$ of infected animals $[4,5]$.

EBL can cause major economic losses to the cattle industry worldwide; it is listed by World Organization for Animal Health (OIE) as a disease of importance to international trade [6]. Many countries have investigated and reported the disease in cattle in recent years $[7,8]$, and the data have supported the prevention and control of EBL to reduce economic losses. However, only one study has reported the seroprevalence of BLV in cattle in northern and northeastern China [9].

Yaks are a unique bovine species and the precious semiwild animal species which lives at high altitudes. Most of them are distributed in the territory of China, especially the white yaks, which only live in the Tianzhu Tibetan Autonomous County (TTAC), Gansu province, northwest China. However, there was no information on the seroprevalence of BLV infection in yaks in China. Therefore, for the first time we surveyed the BLV seroprevalence in yaks in Gansu province, northwest China, and evaluated the risk factors that influenced BLV seroprevalence. 
TABLE 1: Seroprevalence of bovine leukemia virus (BLV) infection in yaks in Gansu province, northwestern China, by enzyme-linked immunosorbent assay (ELISA).

\begin{tabular}{lcccc}
\hline Factor & Category & No. tested & No. positive & $\begin{array}{c}\text { Prevalence } \\
(\%)\end{array}$ \\
\hline \multirow{2}{*}{ Gender } & Male & 471 & 112 & 23.78 \\
& Female & 1113 & 222 & 19.95 \\
\hline \multirow{4}{*}{ Age (years) $)$} & $1<$ years $\leq 3$ & 594 & 123 & 19.93 \\
& $3<$ years $\leq 5$ & 355 & 89 & 20.71 \\
& $5<$ years & 349 & 65 & 25.07 \\
\hline \multirow{4}{*}{ Pregnancy } & $\leq 2$ & 854 & 171 & 20.02 \\
& $3-5$ & 189 & 38 & 20.11 \\
& $\geq 6$ & 70 & 13 & 18.57 \\
\hline \multirow{4}{*}{ Season } & Spring & 428 & 99 & 23.13 \\
& Summer & 354 & 70 & 19.77 \\
& Autumn & 467 & 105 & 22.48 \\
\multirow{2}{*}{ Species } & Winter & 335 & 60 & 17.91 \\
\hline \multirow{2}{*}{ Region } & White yaks & 974 & 186 & 19.10 \\
& Black yaks & 610 & 148 & 24.26 \\
\hline \multirow{2}{*}{ Total } & Tianzhu & 974 & 186 & 19.10 \\
& Luqu & 146 & 33 & 22.60 \\
\hline & Maqu & 464 & 115 & 24.78 \\
\hline
\end{tabular}

\section{Materials and Methods}

2.1. Study Area. Serum samples were collected from black and white yaks in various regions in Gansu province $\left(32^{\circ} 31^{\prime \prime}-\right.$ $\left.42^{\circ} 57^{\prime \prime} \mathrm{N}, 92^{\circ} 13^{\prime \prime}-108^{\circ} 46^{\prime \prime} \mathrm{E}\right)$, northwest China. These regions have a plateau continental climate, and the average annual temperatures vary between $-8^{\circ} \mathrm{C}$ and $4^{\circ} \mathrm{C}$.

2.2. Sample Collection. Blood samples were collected between April 2013 and March 2014. A total of 974 blood samples were collected from white yaks randomly from several farms in TTAC, Gansu province, northwest China. A total of 610 blood samples were collected from black yaks randomly from several farms in Luqu and Maqu counties, Gannan Tibetan Autonomous Prefecture, Gansu province, northwest China. All the blood samples were transported directly to the laboratory. Serum was obtained through centrifugation at $1000 \times \mathrm{g}$ for $5 \mathrm{~min}$. The serum was separated and stored at $-20^{\circ} \mathrm{C}$ until analysis. Detailed information regarding breed, geographic origin, season, number of pregnancies, gender, age, and farm were recorded and listed in Tables 1 and 3.

2.3. Serological Assay. Serum samples were examined for antibodies against BLV using a commercially available ELISA kit (Pourquier, Montpellier, France) according to the manufacturer's instructions [10]. Positive and negative controls were provided with the kit. The optical density of color development was read by a photometer at $450 \mathrm{~nm}$, and the titer of antibodies in the sera was determined following the manufacturer's recommendations [10].

2.4. Data Analysis. Seropositive yaks were analyzed in relation to gender, age, number of pregnancies, season, breed, and region. Exploratory analysis was performed to determine variables potentially associated with exposure to BLV. These factors were studied in a multivariable logistic regression model, and if the $(P)$ value was $<0.05$, it was considered a risk factor for BLV infection. The statistical program PASW Statistics 19.0 (SPSS Inc., IBM Corporation, Somers, NY) was used to perform the statistical analysis.

\section{Results and Discussion}

It was demonstrated that $334(21.1 \%)$ out of 1584 serum samples were BLV seropositive, with $24.26 \%$ in black yaks and $19.10 \%$ in white yaks (Table 1 ). The BLV seropositive yaks were found in all herds. BLV seroprevalence ranged from $18.63 \%$ to $25.1 \%$ in different age groups of yaks. The highest and lowest rates were recorded in the $3<$ years $<5$ and $5<$ years old groups, respectively (Table 1 ). Male yaks had a higher seroprevalence $(23.78 \%$ ) compared to females (19.95\%) (Table 1). Among yaks sampled in different seasons, the seroprevalence varied from $17.91 \%$ to $23.13 \%$ (Table 1 ). Among yaks from different regions, BLV seroprevalence ranged from $19.10 \%$ to $24.78 \%$ (Table 1 ). In addition, BLV seroprevalence in female yaks of different pregnancy status ranged from $18.57 \%$ to $20.11 \%$ (Table 1 ). Among the different farms, BLV seroprevalence varied from $16.00 \%$ to $28.16 \%$ (Table 3).

According to conditional forward stepwise logistic regression, it was found that gender, age, region, farm, and number of pregnancies of yaks were not significant risk factors for BLV $(P>0.05)$. The breed of yaks was considered to be a risk factor that influences the BLV seroprevalence in yaks $(P<0.05)$ (Table 2$)$.

In this study, the overall BLV seroprevalence in yaks was $21.09 \%$, which was slightly lower than that reported in dairy cattle $(25.4 \%)$ in Iran [8] but higher than that in breeding beef $(16.3 \%)$ in Japan [11]. The BLV seroprevalence in yaks in this study was much higher than previous reports $[12,13]$. On the other hand, BLV seroprevalence in this study was much lower than the seroprevalence in cattle in Japan (28.6\%) [11], in Ontario in Canada (29.4\%) [14], and in Syria (69.2\%) [15]. Many factors may be contributable to such differences, such as the climatic conditions, geographical conditions, species and farm management, and the sensitivity of the detection techniques used.

Once an animal is infected with BLV, it will remain infected for life. The prevalence determined for the different seasons therefore cannot be considered as a possible risk factor, although the BLV seroprevalence varied from $17.91 \%$ to $23.13 \%$ in different season groups, with slight differences among different seasons, and slightly higher seroprevalences were found in yaks sampled in spring $(23.12 \%)$ and autumn $(22.48 \%)$ (Table 1$)$. This result was in line with the characteristic of BLV that BLV is a persistent infection. 
TABLE 2: Odds ratios for specie as risk factor for bovine leukemia virus (BLV) seroprevalence in yaks $(n=1584)$.

\begin{tabular}{|c|c|c|c|c|c|}
\hline Factor & Group & Prevalence (\%) & OR & $95 \% \mathrm{CI}$ & $P$ value \\
\hline \multirow{2}{*}{ Specie } & White yaks & 19.10 & Reference & & \\
\hline & Black yaks & 24.27 & 1.36 & $1.06-1.73$ & 0.014 \\
\hline
\end{tabular}

TABLE 3: Seroprevalence of bovine leukemia virus (BLV) infection in yaks in different farms in Gansu province, northwestern China.

\begin{tabular}{lcccc}
\hline Region & Farm & No. tested & No. positive & Prevalence (\%) \\
\hline \multirow{4}{*}{ Tianzhu } & 1 & 220 & 51 & 23.18 \\
& 2 & 273 & 51 & 18.68 \\
& 3 & 231 & 44 & 19.05 \\
& 4 & 250 & 40 & 16.00 \\
\hline \multirow{4}{*}{ Maqu } & 5 & 81 & 19 & 23.46 \\
& 6 & 147 & 35 & 23.81 \\
& 7 & 103 & 29 & 28.16 \\
& 8 & 133 & 32 & 24.06 \\
\hline \multirow{4}{*}{ Luqu } & 9 & 60 & 15 & 25.00 \\
& 10 & 34 & 8 & 23.53 \\
Total & 11 & 52 & 10 & 19.23 \\
\hline
\end{tabular}

Moreover, BLV seroprevalences in yaks of different age groups were slightly different, but the difference was not statistically significant $(P>0.05)$. The seroprevalence increased gradually from $19.93 \%$ in years $\leq 1$ age group to $25.07 \%$ in $3<$ years $\leq 5$ age group, and the highest seroprevalence was the $3<$ years $\leq 5$ age group. These results are similar to that of a recent study [16]. These differences may be due to the fact that BLV incubation period is 3-4 years on average. Furthermore, BLV seroprevalences in yaks of different farm groups ranged from $16.00 \%$ to $28.16 \%$. In general, the seroprevalence in farms of TTAC was slightly lower than in Maqu and Luqu counties. But it was not a significant risk factor for BLV $(P>0.05)$. These slight differences could be because TTAC is located near Lanzhou, the capital of Gansu province, and the yak farms are better managed than that in Maqu and Luqu counties.

This study showed that the breed of yaks was the only risk factor associated with BLV seroprevalence. Logistic regression analysis showed that the black yaks had a 1.36 times higher risk of being infected than the white yaks $(\mathrm{OR}=1.36$, 95\% CI $=1.06-1.73$ ). There could be a few explanations for this difference. First, white yaks and black yaks are different yak breeds, and they may have different susceptibility to infection with BLV due to their genetic difference. Second, white yaks get better animal welfare and are better managed because white yak is a unique and precious yak breed, only lives in TTAC, Gansu Province, Northwest China, and total number is only about 50000 . They may have better resistance to infection with BLV than that of black yaks.

This is the first report of BLV seroprevalence in yaks in China. This study extended the host range for BLV. Moreover, the findings of the present study provided baseline information for the timely execution of strategies and measures to control BLV infection in yaks and to assess resulting effects.

\section{Competing Interests}

The authors declare that there are no competing interests regarding the publication of this paper.

\section{Acknowledgments}

This work was supported in part by the "Special Fund for Agro-scientific Research in the Public Interest" (Grant no. 201303037) and the Beef and Yak Production System Programme, MOA (CARS-38).

\section{References}

[1] S. J. Kenyon and C. E. Piper, "Cellular basis of persistent lymphocytosis in cattle infected with bovine leukemia virus," Infection and Immunity, vol. 16, no. 3, pp. 891-897, 1977.

[2] I. Schwartz and D. Lévy, "Pathobiology of bovine leukemia virus," Veterinary Research, vol. 25, no. 6, pp. 521-536, 1994.

[3] G. Gutiérrez, I. Alvarez, R. Politzki et al., "Natural progression of bovine leukemia virus infection in Argentinean dairy cattle," Veterinary Microbiology, vol. 151, no. 3-4, pp. 255-263, 2011.

[4] A. Burny, Y. Cleuter, R. Kettmann et al., "Bovine leukaemia: facts and hypotheses derived from the study of an infectious cancer," Veterinary Microbiology, vol. 17, no. 3, pp. 197-218, 1988.

[5] S. M. Rodríguez, A. Florins, N. Gillet et al., "Preventive and therapeutic strategies for bovine leukemia virus: lessons for HTLV,' Viruses, vol. 3, no. 7, pp. 1210-1248, 2011.

[6] OIE, "Enzootic bovine leukosis," in Manual of Diagnostic Tests and Vaccines for Terrestrial Animals, pp. 729-738, OIE, Paris, France, 6th edition, 2008.

[7] O. Nekouei, J. VanLeeuwen, J. Sanchez, D. Kelton, A. Tiwari, and G. Keefe, "Herd-level risk factors for infection with bovine leukemia virus in Canadian dairy herds," Preventive Veterinary Medicine, vol. 119, no. 3-4, pp. 105-113, 2015.

[8] S. Mousavi, A. Haghparast, G. Mohammadi, and S. E. Tabatabaeizadeh, "Prevalence of bovine leukemia virus (BLV) infection in the northeast of Iran," Veterinary Research Forum, vol. 5, no. 2, pp. 135-139, 2014.

[9] W.-W. Sun, W.-F. Lv, W. Cong et al., "Mycobacterium avium subspecies paratuberculosis and bovine leukemia virus seroprevalence and associated risk factors in commercial dairy and beef cattle in Northern and Northeastern China," BioMed Research International, vol. 2015, Article ID 315173, 7 pages, 2015.

[10] H. Morovati, E. Shirvani, V. Noaman et al., "Seroprevalence of bovine leukemia virus (BLV) infection in dairy cattle in Isfahan Province, Iran," Tropical Animal Health and Production, vol. 44, no. 6, pp. 1127-1129, 2012.

[11] K. Murakami, S. Kobayashi, M. Konishi, K.-I. Kameyama, T. Yamamoto, and T. Tsutsui, "The recent prevalence of Bovine 
Leukemia Virus (BLV) infection among Japanese cattle," Veterinary Microbiology, vol. 148, no. 1, pp. 84-88, 2011.

[12] S. D. Morris, J. G. Myburgh, M. van Vuuren, and F. van der Vyver, "Serological survey to determine the prevalence of bovine leukaemia virus antibodies in dairy cattle on selected farms in the Gauteng and Mpumalanga Provinces," Journal of the South African Veterinary Association, vol. 67, no. 3, pp. 146-147, 1996.

[13] M. Şevik, O. Avcı, and Ö. B. İnce, "An 8-year longitudinal sero-epidemiological study of bovine leukaemia virus (BLV) infection in dairy cattle in Turkey and analysis of risk factors associated with BLV seropositivity," Tropical Animal Health and Production, vol. 47, no. 4, pp. 715-720, 2015.

[14] R. B. Walsh, D. F. Kelton, S. K. Hietala, and T. F. Duffield, "Evaluation of enzyme-linked immunosorbent assays performed on milk and serum samples for detection of neosporosis and leukosis in lactating dairy cows," The Canadian Veterinary Journal, vol. 54, no. 4, pp. 347-352, 2013.

[15] A. Kurdi, P. Blankenstein, O. Marquardt, and D. Ebner, "Serologic and virologic investigations on the presence of BLV infection in a dairy herd in Syria," Berliner und Münchener Tierärztliche Wochenschrift, vol. 112, no. 1, pp. 18-23, 1999.

[16] T. Watanabe, E. Inoue, H. Mori, Y. Osawa, and K. Okazaki, "Delayed-onset enzootic bovine leukosis possibly caused by superinfection with bovine leukemia virus mutated in the pol gene," Archives of Virology, vol. 160, no. 8, pp. 2087-2091, 2015. 


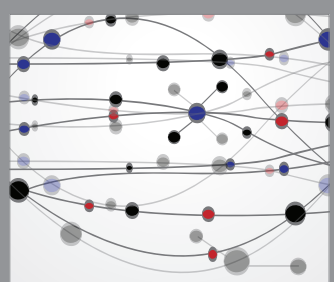

The Scientific World Journal
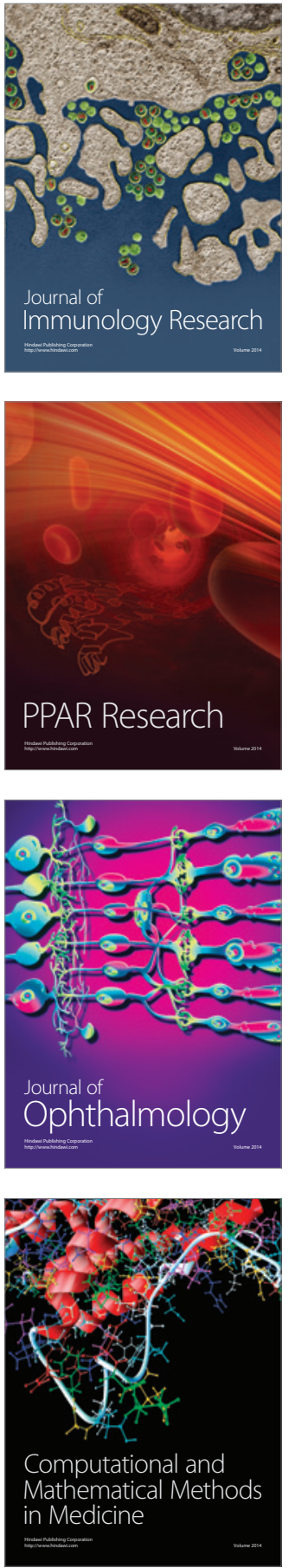

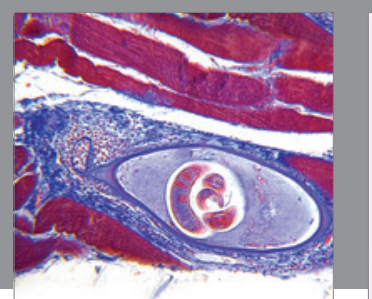

Gastroenterology Research and Practice

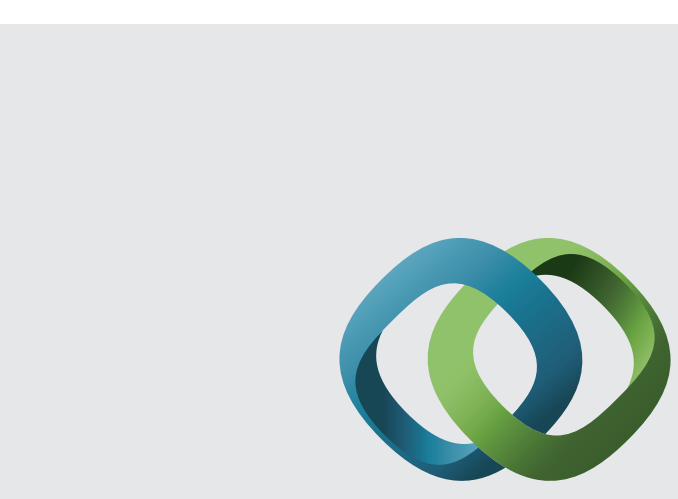

\section{Hindawi}

Submit your manuscripts at

http://www.hindawi.com
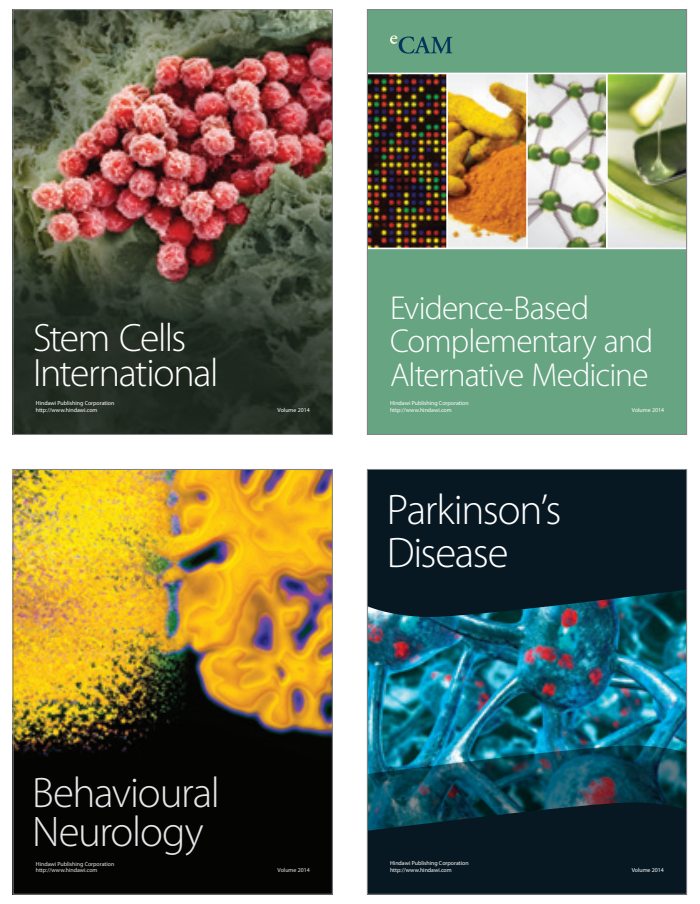
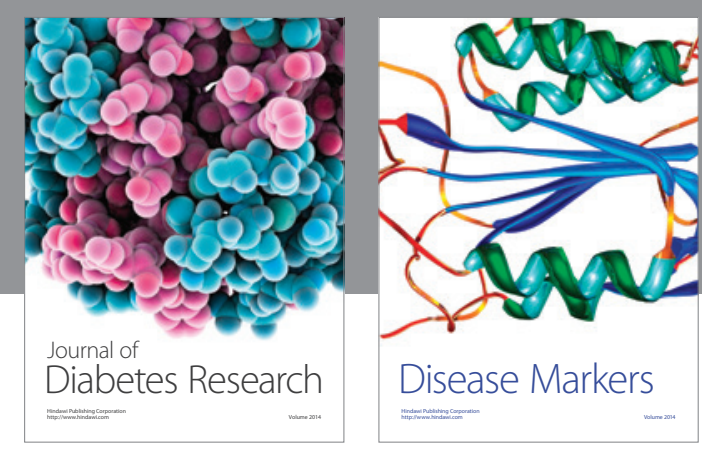

Disease Markers
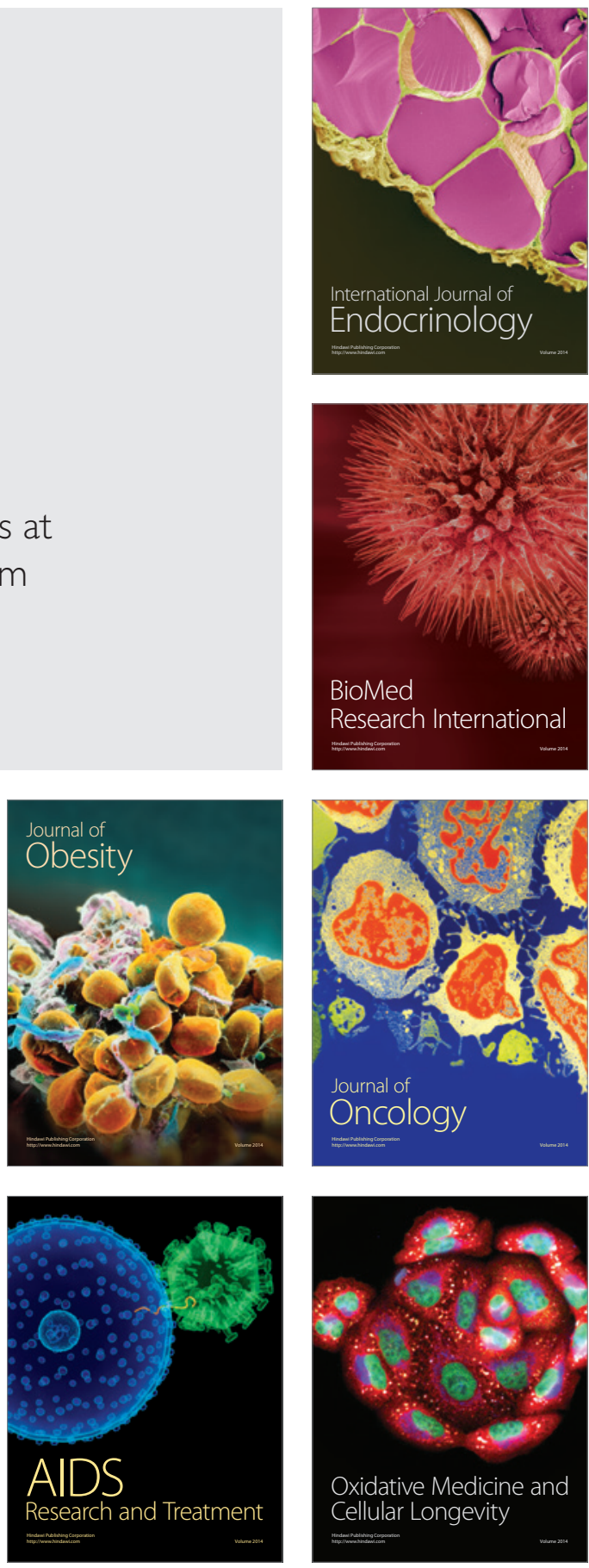